\title{
A Polarization Splitter and Rotator Based on a Partially Etched Grating-assisted Coupler
}

\author{
Ting Hu, Mohamed Saïd Rouifed, Haodong Qiu, Xin Guo, Callum G Littlejohns, Chongyong Liu, and \\ Hong Wang
}

\begin{abstract}
A fabrication-tolerant mid-infrared silicon polarization splitter and rotator (PSR) based on a partially etched grating-assisted coupler is proposed. The design of the partially etched structure allows to use different cladding layers, such as $\mathrm{SiO}_{2}$, to make the device compatible with the metal back-end of line process. Moreover, by using the grating-assisted coupler, the device is no longer limited by the precise requirement of the coupling length and strength as those in its counterparts based on directional couplers. The simulation results show that the PSR can work over a wide spectral range of $50 \mathrm{~nm}$ around the mid-infrared wavelength of $2.5 \mu \mathrm{m}$ with the typical TE-to-TM polarization conversion efficiency of $96.83 \%$, conversion loss of $\mathbf{- 0 . 9 7} \mathrm{dB}$ and polarization crosstalk of $\mathbf{- 2 1 . 4 8} \mathrm{dB}$. The TM-to-TM through insertion loss is around $-0.76 \mathrm{~dB}$. The effects of the fabrication errors are analyzed. The numerical simulation results demonstrate that the device has a good fabrication tolerance larger than $45 \mathrm{~nm}$.
\end{abstract}

Index Terms-Silicon on insulator (SOI), polarization splitter and rotator, grating-assisted coupler, mid-infrared

\section{INTRODUCTION}

$I^{1}$ $\mathrm{N}$ the past decade, silicon photonics has attracted worldwide interest due to its small size, high integration, low cost, power reduction and compatibility with the complementary metal oxide semiconductor (CMOS) fabrication process [1]. As we know, that the compact size of silicon photonic devices is due to the high index contrast structure. This causes large structural birefringence which leads the devices to be polarization sensitivity. This characteristic can be utilized to realize polarization multiplexing devices to improve the bandwidth efficiency in high-speed optical communications [2-3]. On the other hand, some devices can only or would be better to work with one polarization, e.g. multimode interferometers, gratings, ring resonators. For these devices, we need to remove the undesired polarizations or rotate them to the working polarization. Hence, the control and management of

This work was supported by the National Research Foundation of Singapore (NRF-CRP12-2013-04) and NTU-A*Star Silicon Technologies Centre of Excellence.

The authors are with the School of Electrical and Electronic Engineering, Nanyang Technological University, 50 Nanyang Avenue, 639798, Singapore (e-mail: huting@ntu.edu.sg; M-Said.Rouifed@ntu.edu.sg; HDQiu@ntu.edu.sg TGuoXin@ntu.edu.sg; $\quad$ CLittlejohns@ntu.edu.sg; LiuCY@ntu.edu.sg; EWANGHONG@ntu.edu.sg ). polarizations in silicon photonic devices is of great importance [4]. Polarization splitter and rotator (PSR) is a key component used to control and manipulate the polarizations in silicon photonic circuits. Several works have been done to demonstrate the PSR [5-8]. It can be found that most of works are based on the mode coupling scheme in a directional coupler. In such devices, the mode in one waveguide can be coupled to the orthogonal mode with the equivalent effective refractive index in the other waveguide. Other implementations of the PSR by converting fundamental transverse magnetic (TM) mode to the first order transverse electric (TE) mode and then converting the first order TE mode to the fundamental TE mode were also reported [9-10].

In this paper, we propose a PSR on the silicon-on-insulator (SOI) platform based on the mode evolution [11-13] in a partially etched grating-assisted coupling system that operates under mechanism different from the previous works [5-10]. The device is compatible with the metal back-end of line process with high fabrication tolerance. It is not limited by the precise requirement of the coupling length and strength as those in the directional couplers. In addition, the PSR works over a wide spectral range around the mid-infrared wavelength of 2.5 $\mu \mathrm{m}$, with great potential applications in the light detection and ranging (LIDAR), free-space communication and gas sensing systems [14].

\section{DEVICE DESIGN AND Simulation}

The schematic structure of the proposed PSR is illustrated in Fig. 1. It consists of a silicon waveguide A with width $W_{a}$ and height $H$ coupling to a partially etched waveguide $\mathrm{B}$, on the top of the $\mathrm{SiO}_{2}$ box layer. The upper cladding layer is also $\mathrm{SiO}_{2}$, which makes the PSR compatible with the metal back-end of line process. The corrugations are designed beside the sidewall of both two waveguides as the coupling assisted gratings, with the period $\Lambda$, width $W_{\text {cor }}$, period number $N$ and duty cycle $\eta$, respectively. There are three sections in waveguide $\mathrm{B}$. The first section $\left(\mathrm{S}_{1}\right)$ is the partially etched waveguide of width $W_{b 1}$ and height $H_{e}$. The second section $\left(\mathrm{S}_{2}\right)$ is a partially etched taper waveguide with the length of $L_{\text {tap }}$. And the last part $\left(\mathrm{S}_{3}\right)$ is a strip waveguide of width $W_{b 2}$ and height $H$. The four ports of the PSR are denoted from $\mathrm{P}_{1}$ to $\mathrm{P}_{4}$.

The operating principle of the PSR is as follows: Due to the partially etched structure in waveguide $\mathrm{B}$, the co-directional coupling between the two waveguides is suppressed and 
consequently very weak. In the grating-assisted coupling region, the launched fundamental TE mode in waveguide A will be coupled to the first section of waveguide $\mathrm{B}$ at the phase matching condition $\lambda=\Lambda \cdot\left(n_{A}+n_{B}\right)$, where $n_{A}$ and $n_{B}$ represent the TE-mode effective indices of waveguides $\mathrm{A}$ and $\mathrm{B}, \lambda$ is the Bragg wavelength. Then it is converted to the TM mode in the following two sections of waveguide B. On the other hand, if the fundamental TM mode is launched into waveguide A, it will transmit to port $\mathrm{P}_{3}$ directly.
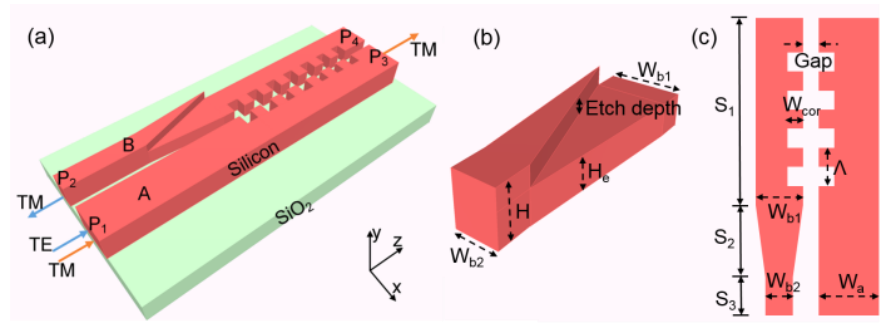

Fig. 1. Schematic configuration of the proposed PSR, (a) the 3D view, (b) the zoom in of the partially etched taper, and (c) the top view. The upper cladding layer is not shown here for clarity.

The three dimensional finite difference time domain (3D FDTD) method is employed to simulate the proposed PSR. In order to evaluate the polarization conversion efficiency (PCE), the normalized TE and TM polarization transmission power are calculated by the integration of the Poynting vector in the cross-section surface. In our design, the optimal structure parameters are chosen as: $W_{a}=0.7 \mu \mathrm{m}, W_{b 1}=0.6 \mu \mathrm{m}, W_{b 2}=0.4$ $\mu \mathrm{m}, H=0.6 \mu \mathrm{m}, H_{e}=0.4 \mu \mathrm{m}, L_{\text {tap }}=30 \mu \mathrm{m}, G a p=0.18 \mu \mathrm{m}, \Lambda=0.56$ $\mu \mathrm{m}, W_{c o r}=0.15 \mu \mathrm{m}, N=80, \eta=50 \%$. The refractive indices of $\mathrm{Si}$ and $\mathrm{SiO}_{2}$ are set as 3.442 and 1.429 with the imaginary part of zero for wavelengths around $2.5 \mu \mathrm{m}$ [15], which means the absorption loss is negligible. The simulation results, as presented in Fig. 2, prove that the proposed PSR realizes the beam splitting and rotating efficiently. When the fundamental TE mode is launched into port $P_{1}$, it is gradually contra-directional coupled to the first section of waveguide $\mathrm{B}$ and then converted to TM mode and drop at port $\mathrm{P}_{2}$, as seen in Fig. 2(a). The corresponding electric field of $E_{x}$ and $E_{y}$ at port $\mathrm{P}_{2}$ are shown in Fig. 2(c) and 2(d), from which we can see that the $\mathrm{E}_{\mathrm{y}}$, or said as TM polarization, is the absolutely dominant component rather than TE polarization. For the other condition, if the fundamental TM mode is sent into port $\mathrm{P}_{1}$, as shown in Fig. 2(b), it will transmit to port $\mathrm{P}_{3}$ directly. The corresponding $E_{x}$ and $E_{y}$ at port $P_{3}$ are shown in Fig. 2(e) and 2(f). Obviously, the major component is the TM polarization.

A further quantitative illustration of the PSR function is realized by sweeping the working wavelengths to obtain the TE and TM polarization transmission spectra at $\mathrm{P}_{2}$ and $\mathrm{P}_{3}$. The simulation results are plotted in Fig. 3. The TE and TM polarization transmission at port $\mathrm{P}_{2}$ (magenta dashed line for TE, magenta solid line for TM) and $\mathrm{P}_{3}$ (blue dashed line for TE, blue solid line for TM) with the TE mode launched into $\mathrm{P}_{1}$ are shown in Fig. 3(a). As seen from the figure, for a wide range of $50 \mathrm{~nm}$ from wavelength 2.475 to $2.525 \mu \mathrm{m}$, most of the input light (around $80 \%$, an equivalent insertion loss (IL) of $-0.97 \mathrm{~dB}$ ) is coupled to port $\mathrm{P}_{2}$, and in which the TM polarization is the absolute majority. The TE-to-TM PCE, calculated as $P C E=P_{T M} /\left(P_{T M}+P_{T E}\right) \times 100 \%$, is over $96.83 \%$ in this spectral range. Fig. 3(b) shows the TE and TM polarization transmission at port $\mathrm{P}_{2}$ and $\mathrm{P}_{3}$ under the TM mode launched condition. It can be seen that, beyond the wavelength $2.47 \mu \mathrm{m}$, the input light is transmitted to port $\mathrm{P}_{3}$ directly, and the dominant component is the TM polarization. The typical $I L$ for TM-to-TM through transmission between port $\mathrm{P}_{1}$ and $\mathrm{P}_{3}$ from 2.475 to $2.525 \mu \mathrm{m}$ is around $-0.76 \mathrm{~dB}$. The typical crosstalk (CT) from $\mathrm{P}_{2}$ to $\mathrm{P}_{3}$ under the TE mode input condition, and the $C T$ from $\mathrm{P}_{3}$ to $\mathrm{P}_{2}$ under the TM mode input situation could also be extracted. They are -21.48 and $-18.98 \mathrm{~dB}$, respectively. The polarization transmission (olive dashed line for TE, olive solid line for $\mathrm{TM}$ ) of the reflection light at port $\mathrm{P}_{1}$ are plotted as well in Fig. 3(a) and 3(b) to evaluate the optical power loss of the device. When TE mode is launched into $\mathrm{P}_{1}$, as seen in Fig. 3(a), the dominant polarization of the reflection light at $\mathrm{P}_{1}$ is $\mathrm{TM}$, which indicates that the optical power is coupled from $\mathrm{P}_{2}$. The optical power of the back transmission at $\mathrm{P}_{1}$ is very low $(\sim 0.092)$. The sum of normalized power of $\mathrm{P}_{1}, \mathrm{P}_{2}$ and $\mathrm{P}_{3}$ extracted from Fig. 3(a) is around 0.937, which suggests that about $6.3 \%$ of power losses induced by scattering in the grating-assisted coupling process since the absorption loss is negligible as mentioned before. For the TM mode input, the scattering loss calculated from Fig. 3(b) is about 7.9\%.
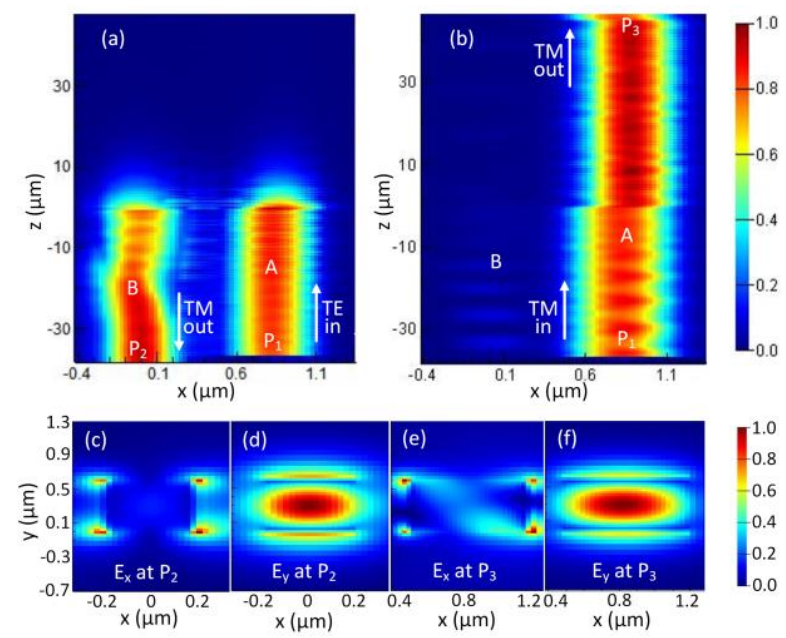

Fig. 2. (a) Light propagation in the $x z$ plane of the PSR, electric field of (c) $E_{x}$ and (d) $E_{y}$ at port $P_{2}$ when the fundamental TE mode is launched. (b) Light propagation in the xz plane of the PSR, electric field of (e) $E_{x}$ and (f) $E_{y}$ at port $\mathrm{P}_{3}$ when the fundamental TM mode is launched.

In fact, the performance of PSR can be tailored with the trade-off between the spectral range and $I L$ for different application requirements. For example, a PSR with lower $I L$ is needed, a smaller-corrugated grating can be used with the sacrifice of working bandwidth (WB). Fig. 3(c) and 3(d) show the simulation results of the PSR with smaller corrugation of $W_{\text {cor }}=0.1 \mu \mathrm{m}(\Lambda=0.54 \mu \mathrm{m}$, the other structure parameters are the same with that used to calculate Fig. 3(a) and 3(b)). As seen, compared to the simulation results of $W_{c o r}=0.15 \mu \mathrm{m}$, the TE-to-TM conversion loss and TM-to-TM transmission loss 
decrease to $-0.71 \mathrm{~dB}(\sim 85 \%$ transmission $)$ and $-0.22 \mathrm{~dB}(\sim 95 \%$ transmission), respectively. However, the sacrifice is that the WB shrinks to $\sim 26.5 \mathrm{~nm}$. The scattering loss extracted from Fig. $3(\mathrm{c})$ and $3(\mathrm{~d})$ is about $1.4 \%$ and $0.2 \%$ for TE and TM mode input, respectively. It is worth to mention that the proposed PSR also can work in other wavelengths by redesign the dimensional parameters, such as the period $\Lambda$. However, if the working wavelength extends to $3.5 \mu \mathrm{m}$ and beyond, the absorption loss of $\mathrm{SiO}_{2}$ is not negligible. Thus, other cladding materials such as $\mathrm{Si}_{3} \mathrm{~N}_{4}$ could be considered. The SOI platform with $\mathrm{SiO}_{2}$ cladding is used for our device because it is commercially available, and the absorption loss is negligible at the working wavelength around $2.5 \mu \mathrm{m}$.
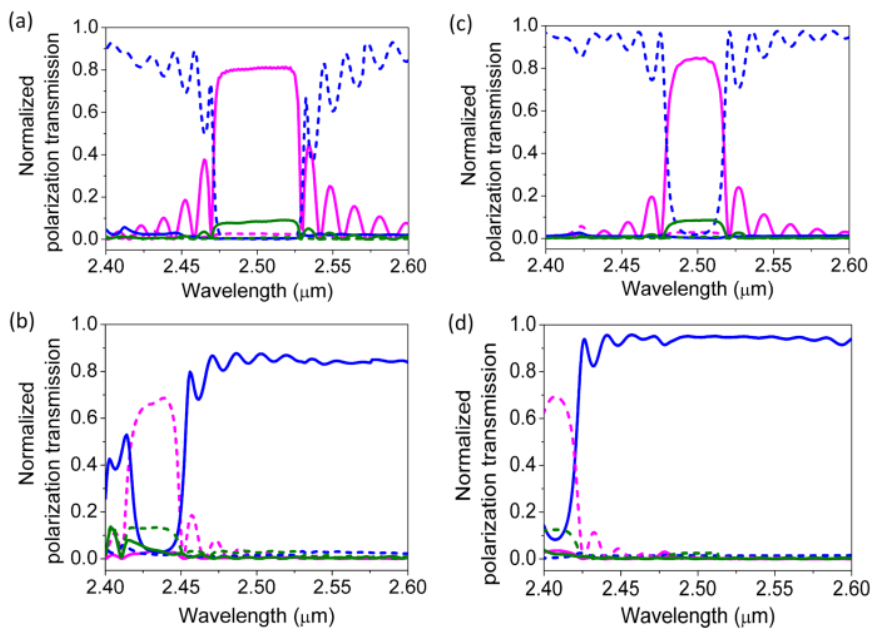

Fig. 3. Normalized polarization transmission at port $\mathrm{P}_{1}$ (olive line), $\mathrm{P}_{2}$ (magenta line) and $\mathrm{P}_{3}$ (blue line) when (a) fundamental TE mode (b) fundamental TM mode are launched into port $\mathrm{P}_{1}$ with corrugation of $W_{c o r}=0.15 \mu \mathrm{m}$, and the (c) fundamental TE mode (d) fundamental TM mode are launched into port $P_{1}$ with corrugation of $W_{c o r}=0.1 \mu \mathrm{m}$. The dashed and solid lines represent the TE and TM polarization, respectively.

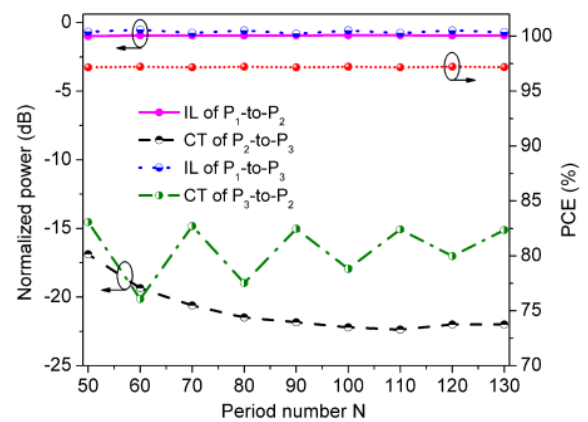

Fig. 4. $I L, C T$ and $P C E$ of the PSR varying with the grating period number $N$ (the coupling length $L=\Lambda \cdot N$ ) at the working wavelength of $2.5 \mu \mathrm{m}$.

Another advantage of the PSR is that the device performance is not limited by the precise control of coupling length as those in the directional couplers. Fig. 4 shows the simulation results of the $I L, C T$ and $P C E$ as functions of the grating period number $N$ (the coupling length $L=\Lambda \cdot N$ ) varying from 50 to 130 with the working wavelength of $2.5 \mu \mathrm{m}$. As can be seen, when the TE mode is launched into port $\mathrm{P}_{1}$, the light is transmitted to port $\mathrm{P}_{2}$ with the $I L$ (magenta solid line) around $-0.94 \mathrm{~dB}$ and the TE-to-TM PCE (red short dot line) around 97.2\%. The worst
$C T$ to port $\mathrm{P}_{3}$ (black dashed line) is $-16.88 \mathrm{~dB}$ when $N=50$. The crosstalk decreases gradually as $N$ increases. When the TM mode is launched into port $\mathrm{P}_{1}$, it propagates to port $\mathrm{P}_{3}$ directly with the $I L$ (blue dot line) around $-0.646 \mathrm{~dB}$. The $C T$ to $\mathrm{P}_{2}$ (olive dashed dot line) fluctuates between -14.54 and -21.48 dB. The unstable $C T$ is caused by the sidelobe changing with different period number $N$.

\section{ANALYSIS OF FABRICATION TOLERANCES}

The fabrication of the designed PSR is fully compatible with the CMOS process. Usually, fabrication errors could be induced during the lithography and other process steps, which result in the variations of the critical dimension (CD). First, the impact of the variations of gap spacing ( $\triangle$ Gap) and partially etched depth $\left(\triangle D_{e}\right)$ on $P C E, I L$ and $C T$ is numerically analyzed. The operating wavelength is also assumed as $2.5 \mu \mathrm{m}$. The simulated result of gap spacing variation is shown in Fig. 5(a). As seen, for a wide variation range from -60 to $80 \mathrm{~nm}$, the device keeps a high PCE around $97.01 \%$, and a low mode conversion loss (IL of $\mathrm{P}_{1}$-to- $\mathrm{P}_{2}$ ) below $-0.97 \mathrm{~dB}$. The $C T$ of $\mathrm{P}_{2}$-to- $\mathrm{P}_{3}$ increases with the gap spacing, however, it is still below $-14.3 \mathrm{~dB}$. For the TM mode launched condition, the $I L$ of $\mathrm{P}_{1}$-to- $\mathrm{P}_{3}$ is around $-0.58 \mathrm{~dB}$, while the $C T$ of $\mathrm{P}_{3}$-to- $\mathrm{P}_{2}$ is below $-14 \mathrm{~dB}$. As aforementioned, the fluctuation of $C T$ from $\mathrm{P}_{3}$-to- $\mathrm{P}_{2}$ is caused by the undesirable sidelobe of other grating filter spectrum adjacent to the working wavelength window of the PSR. The similar phenomenon can be found in Fig. 5(b)-5(g), but they do not prevent the device working efficiently. Notice that the IL has a very slight variation when the gap changes from -60 to $80 \mathrm{~nm}$. It conveys that the proposed PSR is not limited by the precise requirement of the coupling strength (spacing between two waveguides) as those in its counterparts based on directional couplers. Considering the partially etched process, the inaccuracy of the etch depth is analyzed. As shown in Fig. 5(b), for a $\pm 30 \mathrm{~nm}$ deviation, the PSR maintains the PCE higher than $95.86 \%$ and mode conversion loss lower than -0.94 $\mathrm{dB}$. The TM mode transmission loss $\left(I L\right.$ of $\mathrm{P}_{1}$-to- $\left.\mathrm{P}_{3}\right)$ is below $-0.94 \mathrm{~dB}$ with the $C T$ of $\mathrm{P}_{3}$-to- $\mathrm{P}_{2}$ below $-13.91 \mathrm{~dB}$.

Fig. 5(c) shows the influence of waveguide width variation on the PSR performance. When the width of waveguide A varies from -25 to $40 \mathrm{~nm}$, the device remains the $P C E$ over $96.8 \%$, and the $I L$ of $\mathrm{P}_{1}$-to- $\mathrm{P}_{2}$ and $C T$ of $\mathrm{P}_{2}$-to- $\mathrm{P}_{3}$ below -0.94 and $-14.02 \mathrm{~dB}$, respectively. While the TM mode transmission loss is below $-0.8 \mathrm{~dB}$ with the $C T$ of $\mathrm{P}_{3}$-to- $\mathrm{P}_{2}$ below $-13.21 \mathrm{~dB}$. For the variation of the width for waveguide $\mathrm{B}$, as observed in Fig. 5(d), the PSR is able to realize the polarization splitting and rotating in a large $\Delta W_{b}$ span (-30 to $35 \mathrm{~nm}$ ) with the worst $P C E$ of $95.75 \%$, mode conversion loss of $-1.3 \mathrm{~dB}$, TM mode transmission loss of $-0.73 \mathrm{~dB}$, and $C T$ of $-12.29 \mathrm{~dB}$. The variation of device height is also considered. As seen in Fig. $5(\mathrm{e})$, in a wide varying range of $85 \mathrm{~nm}(-60$ to $25 \mathrm{~nm})$, the device keeps the PCE over $96.31 \%$ with the $I L$ below -0.94 and CT below $-12.48 \mathrm{~dB}$. 

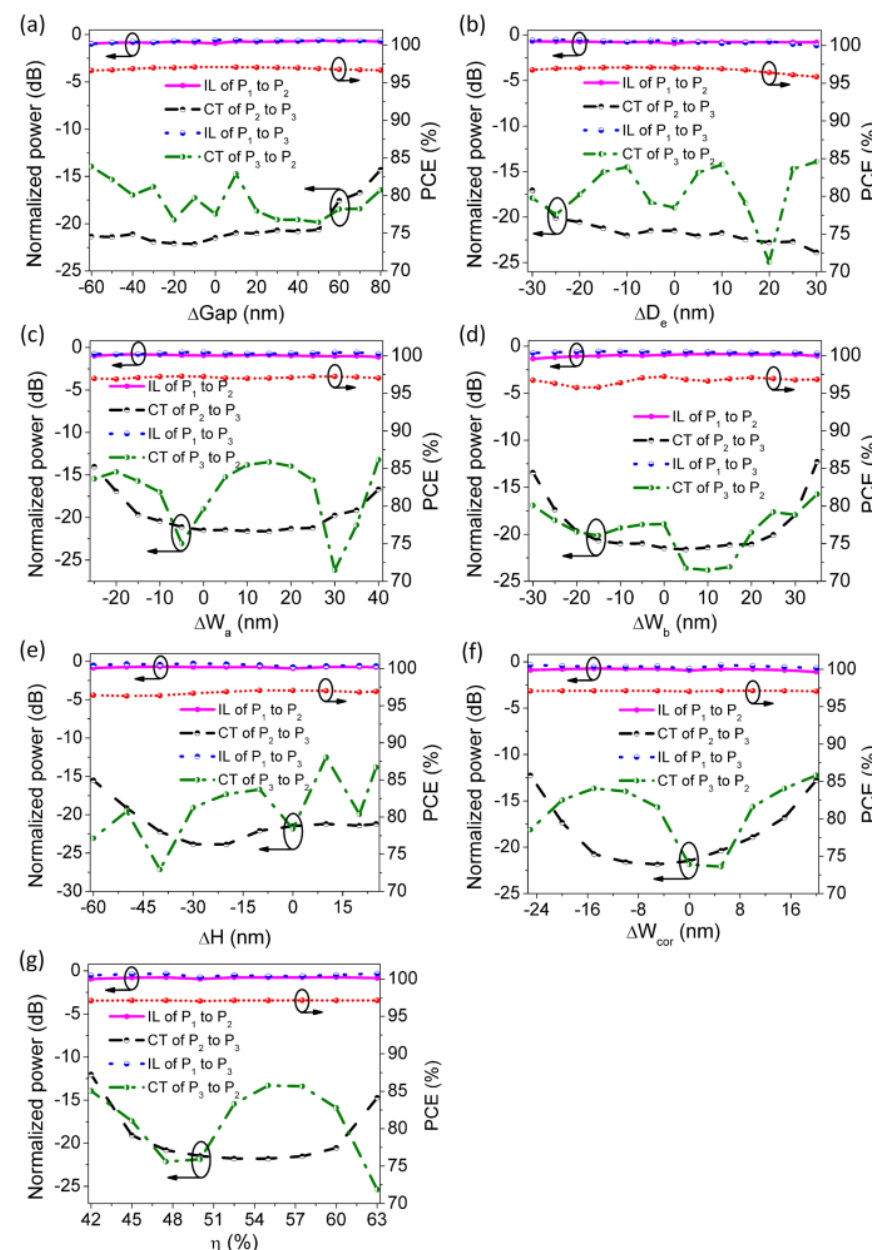

Fig. 5. IL, CT and PCE of the PSR varying with (a) $\triangle G a p$ (b) $\Delta D_{e}$ (c) $\triangle W_{a}$ (d) $\Delta W_{b}$ (for both $W_{b l}$ and $W_{b 2}$ ) (e) $\Delta H$ (f) $\Delta W_{c o r}$ and (g) duty cycle $\eta$ at the working wavelength of $2.5 \mu \mathrm{m}$. The $I L$ of $\mathrm{P}_{1}$ to $\mathrm{P}_{2}, C T$ of $\mathrm{P}_{2}$ to $\mathrm{P}_{3}$ and the $I L$ of $\mathrm{P}_{1}$ to $\mathrm{P}_{3}$, $C T$ of port $\mathrm{P}_{3}$ to $\mathrm{P}_{2}$ are under the TE mode and TM mode launched condition, respectively.

The corrugation width and duty cycle are critical dimensions of the gratings. Due to the dense structure, these dimensions usually shift from the design value in the fabrication process, especially in the exposure steps using electron beam lithography (EBL). Hence the numerically analysis of the performance variation caused by dimension shift of them are carried out. Fig. 5(f) shows that the PSR can work efficiently with high PCE ( 97.1\%) even the corrugation width of $W_{c o r}$ varies from -25 to $20 \mathrm{~nm}$. The TE-to-TM conversion loss is around $-0.77 \mathrm{~dB}$ while the $C T$ of $\mathrm{P}_{2}$-to- $\mathrm{P}_{3}$ is below $-12.26 \mathrm{~dB}$. The TM-to-TM transmission loss is about $-0.48 \mathrm{~dB}$ with an induced $C T$ of $\mathrm{P}_{3}$-to- $\mathrm{P}_{2}$ below $-12.25 \mathrm{~dB}$. From Fig. 5(g) one can see that, the device keeps a high PCE over $97.05 \%$ in a wide duty cycle range from $42 \%$ to $63 \%$ (equivalent to the $117.6 \mathrm{~nm}$ shrink of the trench width in the grating). The worst TE-to-TM conversion/TM-to-TM transmission loss and $C T$ are -0.94 and $-12.03 \mathrm{~dB}$, respectively. The results suggest that the PSR has a large process tolerance over $45 \mathrm{~nm}$, while maintains good performance.

\section{CONCLUSION}

In this paper, a mid-infrared PSR on the SOI is proposed.
The device operates under a mechanism which is different from previous works and it is fully compatible with the metal back-end of line process. The 3D FDTD simulation results show that the proposed PSR is able to work over a wide spectral range of $50 \mathrm{~nm}$ around the wavelength of $2.5 \mu \mathrm{m}$ with the typical TE-to-TM $P C E$ of $96.83 \%$, conversion loss of $-0.97 \mathrm{~dB}$, and polarization crosstalk of $-21.48 \mathrm{~dB}$. A TM-to-TM through $I L$ around $-0.76 \mathrm{~dB}$ is also obtained. Further analysis indicates that the PSR has a large fabrication tolerance over $45 \mathrm{~nm}$. It can be fabricated using CMOS process with great potential applications in the mid-infrared systems.

\section{REFERENCES}

[1] J. J. K. Doylend and A. P. Knights, "The evolution of silicon photonics as an enabling technology for optical interconnection," Laser Photonics Rev., vol. 6, pp. 504-525, 2012.

[2] C. R. Doerr, P. J. Winzer, Y. K. Chen, S. Chandrasekhar, M. S. Rasras, L. Chen, T. Y. Liow, K. W. Ang, and G. Q. Lo, "Monolithic polarization and phase diversity coherent receiver in silicon," J. of Lightwave Technol., vol. 28, pp. 520-525, 2010.

[3] P. Dong, C. Xie, L. Chen, L. L. Buhl, and Y.-K. Chen, "112-Gb/s monolithic PDM-QPSK modulator in silicon,” Opt. Express, vol. 20, pp. B624-B629, 2012.

[4] D. Dai, L. Liu, S. Gao, D. X. Xu, and S. He, "Polarization management for silicon photonic integrated circuits," Laser Photon. Rev., vol. 7, pp. 303-328, 2013.

[5] D. Dai and J. E. Bowers, "Novel concept for ultracompact polarization splitter-rotator based on silicon nanowires," Opt. Express, vol. 19, pp. 10940-10949, 2011.

[6] J. Wang, B. Niu, Z. Sheng, A. Wu, X. Wang, S. Zou, M. Qi, and F. Gan, "Design of $\mathrm{a} \mathrm{SiO}_{2}$ top-cladding and compact polarization splitter-rotator based on a rib directional coupler," Opt. Express, vol. 22, pp. 4137-4143, 2014.

[7] Y. Xiong, J. G. Wangüemert-Pérez, D. X. Xu, J. H. Schmid, P. Cheben, and W. N. Ye, "Polarization splitter and rotator with subwavelength grating for enhanced fabrication tolerance," Opt. Lett., vol. 39, pp. 6931-6934, 2014.

[8] Y. Xiong, D. X. Xu, J. H. Schmid, P. Cheben, S. Janz, and W. N. Ye, "Fabrication tolerant and broadband polarization splitter and rotator based on a taper-etched directional coupler," Opt. Express, vol. 22, pp. 17458-17465, 2014.

[9] Y. Ding, H. Ou, and C. Peucheret, "Wideband polarization splitter and rotator with large fabrication tolerance and simple fabrication process," Opt. Lett., vol. 38, pp. 1227-1229, 2013.

[10] W. D. Sacher, T. Barwicz, B. J. Taylor, and J. K. Poon, "Polarization rotator-splitters in standard active silicon photonics platforms," Opt. Express, vol. 22, pp. 3777-3786, 2014.

[11] J. Zhang, M. Yu, G. Lo, and D.-L. Kwong, "Silicon waveguide-based mode-evolution polarization rotator," IEEE J. Sel. Top. Quantum Electron., vol. 16, pp. 53-60, 2010.

[12] L. Chen, C. R. Doerr, and Y.-K. Chen, "Compact polarization rotator on silicon for polarization-diversified circuits," Opt. Lett., vol. 36, pp. 469471, 2011.

[13] H. J. Zhang, S. Das, Y. Huang, C. Li, S. Y. Chen, H. F. Zhou, M. B. Yu, P. G. Q. Lo, and J. T. L. Thong, "Efficient and broadband polarization rotator using horizontal slot waveguide for silicon photonics," Appl. Phys. Lett., vol. 101, pp. 021105, 2012.

[14] R. Soref, "Mid-infrared photonics in silicon and germanium," Nat. Photonics, vol. 4, pp. 495-497, 2010.

[15] E. D. Palik, Handbook of Optical Constants of Solids. Maryland, Elsevier, 1997, pp. 749-763. 